\title{
Adult emergency department visits during the COVID-19 pandemic in Veneto region, Italy: a time-trend Analysis
}

\author{
Matteo Paganini ${ }^{1,2}$ (1) $\cdot$ Claudio Barbiellini Amidei ${ }^{3} \cdot$ Maria Francesca Valastro $^{2} \cdot$ Andrea Favaro $^{2} \cdot$ Mario Saia $^{4}$. \\ Alessandra Buja ${ }^{3}$
}

Received: 13 July 2021 / Accepted: 21 July 2021 / Published online: 30 July 2021

(c) Società Italiana di Medicina Interna (SIMI) 2021

Keywords Emergency department $\cdot$ Emergency medicine $\cdot$ COVID-19 $\cdot$ Pandemic $\cdot$ Policy

Dear Editor,

In 2020, emergency medical care-as the frontline of healthcare-had to rapidly adapt staff [1], stuff [2], and structures [3] to receive and take care of both suspected and confirmed COVID-19 patients while protecting healthcare workers and continuing to serve the community. Despite large volumes of patients being analyzed, different emergency department (ED) admission patterns have been described in literature during epidemics or pandemics. For example, a decrease in ED total visits has been consistently reported during the 2003 SARS outbreak [4], while a significantly higher number of admissions was registered during influenza seasons (due to influenza-like symptoms) [5]. During the COVID19 pandemic, a consistent decrease in ED visits has been recorded worldwide. Italy has been the first western country to register a reduction in individuals seeking medical attention in EDs, although studies describing this phenomenon are mostly limited to the first pandemic wave [6-8].

To analyze ED visits in the Veneto Region (Northeastern Italy) during the first and second pandemic waves, anonymized records of ED presentations in 2019 and 2020

Matteo Paganini

matteo.paganini@unipd.it

1 Department of Biomedical Sciences, University of Padova, Via Marzolo 3, 35131 Padova, Italy

2 Emergency Department and Emergency Medical Service, "San Bassiano" Hospital, ULSS 7 "Pedemontana", Via dei Lotti, 40, 36061 Bassano del Grappa, Italy

3 Department of Cardiologic, Vascular and Thoracic Sciences, and Public Health, University of Padova, Via Loredan 18, 35131 Padova, Italy

4 Clinical Governance Unit, Azienda Zero, Veneto Region, Pass. Luigi Gaudenzio 1, 35131 Padova, Italy of adults ( $\geq 15$ years) were collected from the regional database of emergency medical care. Veneto Region provides healthcare to an overall population of nearly 4.9 million inhabitants. Overall, 54 EDs were available to the population during the study period -46 public and 8 private. The database contains demographic details (age, gender), date of visit, arrival mode ("walk-in" or "ambulance"), clinical presentation (chief complaint), triage code, and outcome (final disposition). Treatment priority was defined at the moment of presentation by a specifically trained nurse (as per Italian guidelines) attributing one of the following triage codes: white (non-urgent); green (low priority); yellow (urgent, potentially life-threatening condition); red (critical, life-threatening condition). Finally, the outcome of each ED presentation was classified as: death at the ED; hospitalization; home discharge (including referral to ambulatory services for follow-up and refusal of admission or further medical assistance). The trend of monthly ED visits in 2020 was plotted against the new daily SARS-CoV-2 infections and hospitalized COVID-19 patients in Veneto. The bimestrial trend of ED visits for the ten most frequent clinical presentations (over the entire study period) was then graphically represented. A year-over-year change (comparing the same month in 2019 and 2020) in the number of monthly ED visits was calculated, stratified by triage code. Finally, the proportion of visits by arrival mode, triage code, and outcome were calculated for 2019 and 2020. All analyses were carried out with R Studio Integrated Development for R (RStudio, Inc., Boston, MA).

A total of 3,298,672 ED presentations were retrieved. After removing patients aged $<15$ years, $1,665,895$ presentations in 2019 and 1,202,872 in 2020 were included in the study. Gender and age were almost equally distributed between the 2 years. ED visits consistently dropped in March (- 52\%) and April (- 52\%) 2020 during the first pandemic 
wave as already described in other Italian settings [6-8]. During summer, the reduction of SARS-CoV-2 contagion and COVID-19 related-hospitalization rates were associated with a gradual increase in ED presentations. Starting in October 2020, the second wave resulted in a further decline of ED visits, which was more evident in November and December 2020, but less marked than during the first wave (Fig. 1).

Several factors may have contributed to emergency medical care avoidance. Since the Italian National Health Service is a publicly funded, universal healthcare system, requiring co-payment for medical examination and procedures performed in the ED only in specific cases, the fear of unexpected medical bills in this context unlikely explains the phenomenon. No limitations were imposed to impede the population's access to the emergency medical care system in the Region, except for a temporary closure of the Schiavonia's hospital in which the first domestic COVID-19 cases were detected. Nevertheless, this case alone could not explain the steep reductions observed in March-April. It is reasonable to think that, during the lockdown, travel restrictions and the stop of outdoor and sports activities drastically reduced accidental traumas, as the vast closure of working places probably impacted road or occupational accidents. In fact, burn or traumas registered a considerable reduction $(-29.8 \%)$ between 2019 and 2020 (-62.7\% when comparing March and April alone) (Fig. 2).

In pre-pandemic times, individuals' decisions regarding their health are complex and influenced by numerous factors [9]. On the other hand, during epidemics and pandemics, the anxiety deriving from the fear of contagion incentivizes adaptive mechanisms (e.g., the propensity to social distancing, hand sanitization) but can lead to dangerous behavioral modifications (e.g., social isolation or compulsive actions)
[10]. Persons could have perceived EDs as overcrowded structures, with a heightened risk of SARS-CoV-2 infection. Media portraying hospitals on the brink of collapse and daily reports on skyrocketing contagions with a concurrent contraction of ordinary and intensive care beds availability are likely to have promoted anxiety among citizens, as reported during previous outbreaks. Ferry et al., in a small sample of patients presenting for chest pain in a single ED in Scotland, described a reluctance in seeking medical attention at the beginning of the pandemic due to media reports and SARS-CoV-2 exposure concern [11]. Similar media descriptions, along with communications from national and regional governors, and local administrations, asking the population to stay at home and access the ED only in cases of real need, could also have contributed to generating empathy with a further avoidance of emergency medical care, as explained by social imitation theories. In support of this interpretation, the present study found that white and green triage codes accounted for most ED presentation reduction in 2020 (-30\% and $-32 \%$, respectively) (Fig. 3), mainly represented by dermatological, ENT, ophthalmological, and odontostomatological symptoms.

ED overcrowding is a major public health issue worldwide. It is unclear if the dramatic reduction recorded with non-urgent and less urgent triage codes contributed to excess mortality [12], especially regarding patients who did not seek urgent medical attention. However, no difference before and after the COVID-19 outbreak was found in Regional calls to emergency medical service (EMS) [13], if not an increase compared to the same period of the previous year [14] as confirmed by the findings of the present study (arrival by ambulance: $34.7 \%$ in 2020, $28.6 \%$ in 2019). Also, hospitalizations following the ED visits increased from $15.0 \%$ in 2019 to $21.0 \%$ in 2020 (excluding

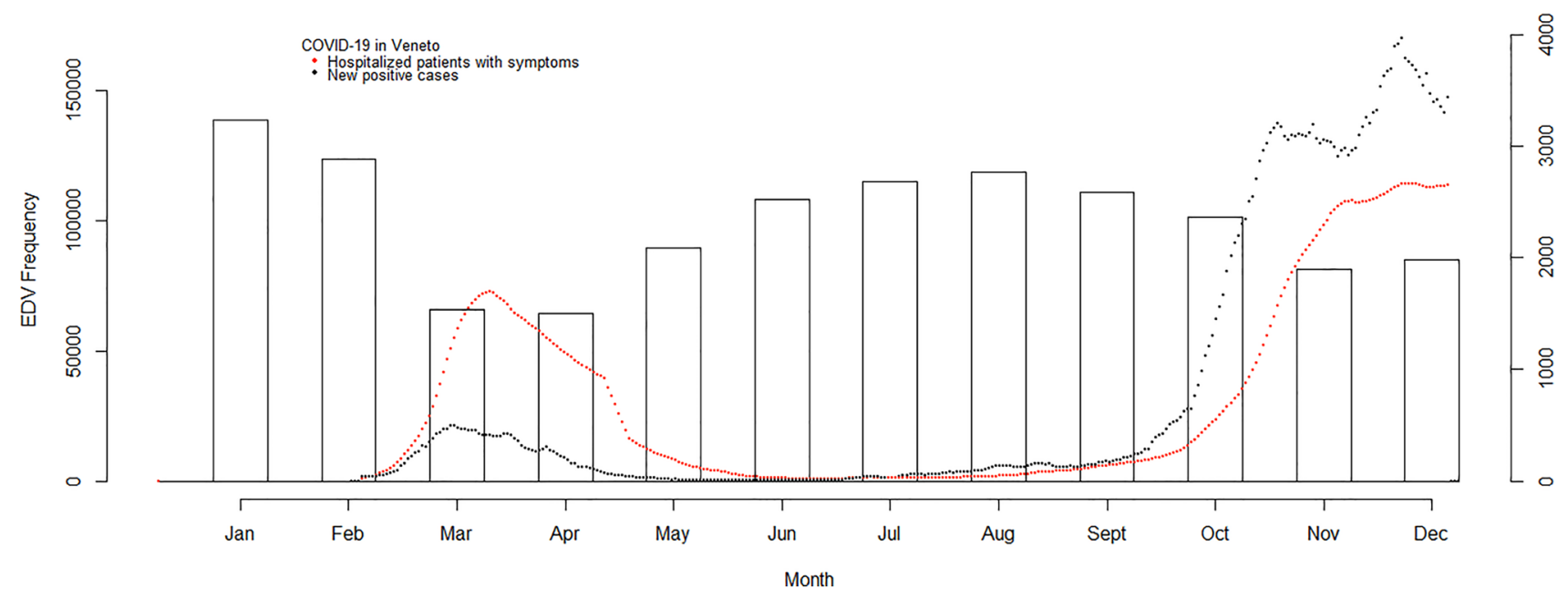

Fig. 1 Emergency Department Visits (EDVs) in the Veneto Region and regional incidence of COVID-19 (daily new cases and COVID-19 hospitalized patients). Presentations significantly dropped in March and April 2020 (-52\% in both months) 


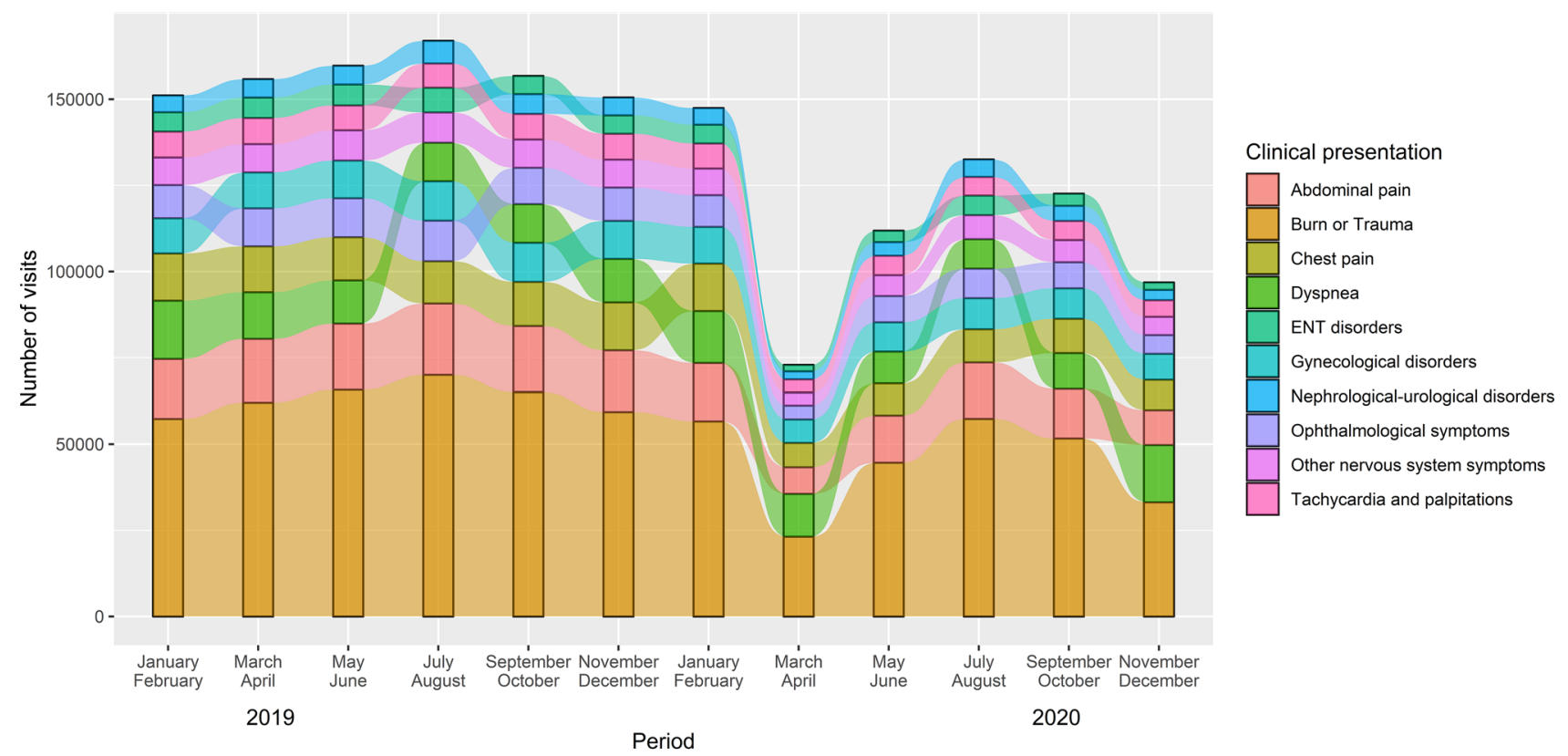

Fig. 2 Bimestrial trend of emergency department visits for the ten most frequent clinical presentations among adults in Veneto Region (2019-2020). The "Burn or Trauma" category represented the most frequent presentation, with a marked drop during the first pandemic wave. Dyspnea as chief complaint instead increased during both pandemic waves
2019

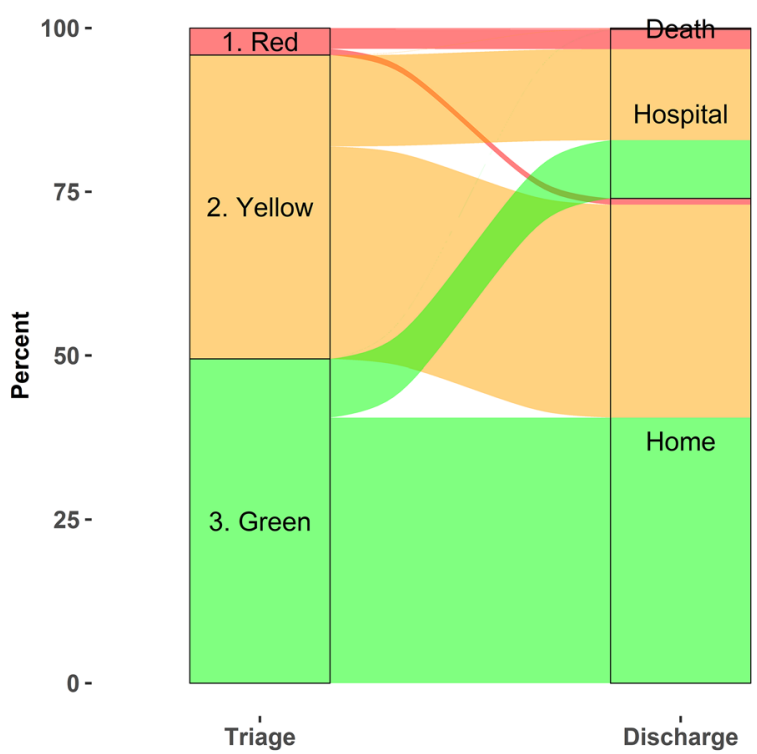

2020

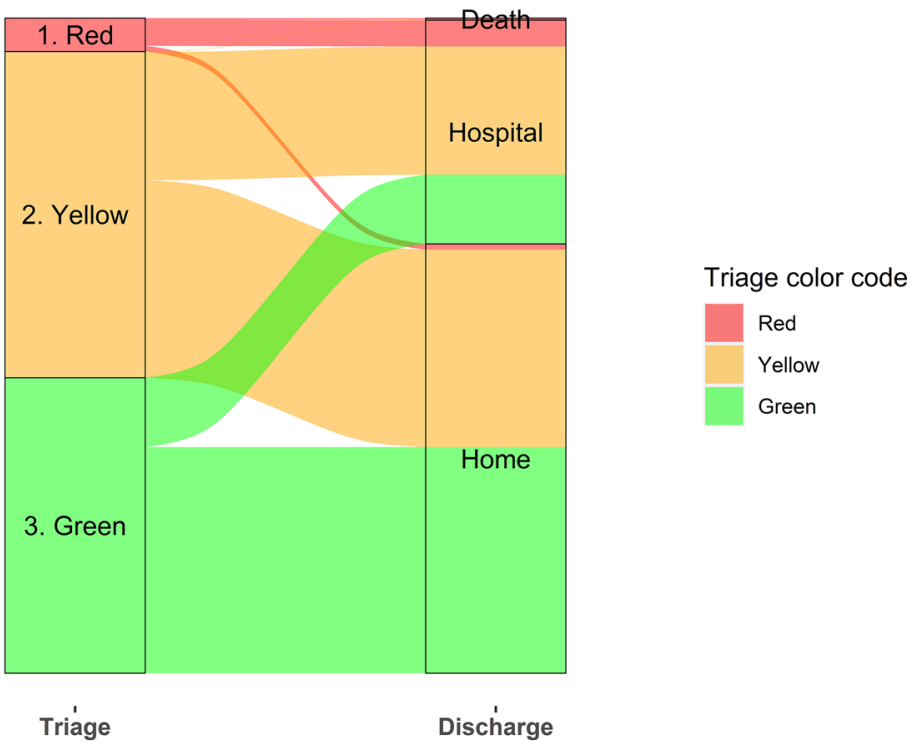

Fig. 3 Triage codes and outcome of emergency department (ED) visits in the Veneto Region (2019-2020). The analysis included 807,080 ED presentations in 2019 and 596,398 in 2020. All white codes were excluded $(N=1,457,515)$. Also, presentations with missing data regarding outcomes of interest were excluded $(N=7774)$ white triage codes: from $25.8 \%$ in 2019 to $34.2 \%$ in 2020). A marked increase can be observed as well in the percentage of yellow triage codes (Fig. 3), with dyspnea becoming the second most common clinical presentation in both pandemic peaks (Fig. 2). This trend suggests that individuals experiencing severe and worrisome symptoms-potentially with an urgent underlying medical condition-sought medical attention or presented any way to the ED and were admitted. Yet, looking into the reasons for admission goes beyond the purpose of this study, and as such, a similar 
conclusion will require further studies to be confirmed, and so will trends concerning other reasons for admissions. What is clear is that low priority codes dropped, potentially reducing overcrowding and unnecessary access to EDs. The consequences on healthcare use deriving from the present pandemic can allow us to tackle the increasingly recognized challenge of "too much medicine", when unnecessary ED visits end up being harmful to the individual and the society by consuming unnecessary resources [15]. A future integrated approach based on the national registry-reporting specific causes of mortality-and the analysis of specific conditions and symptoms that underwent the greatest reduction among ED admissions could be attempted to provide useful implications for both emergency medicine and public health.

It was impossible to detect repeated visits to the ED or further adjust for comorbidities or other individual characteristics since data retrieved from the regional database were anonymized. However, the large population and the presence of a public healthcare system allowed to obtain an unbiased overview of ED visit trends.

In conclusion, this is the first population-based study in Italy to examine in-depth ED presentations during the first two COVID-19 pandemic waves and trace patients' specific comparative trajectories. These findings suggest a drastic reduction of non-urgent and less urgent ED presentations that are likely to be reconducted to lockdown measures and perceived fear of contagion in hospitalbased services. Given how relevant appropriateness in emergency medical care is, future studies should shed light on the actual causes and the consequences of the drastic reduction in ED visits observed in the present study. Such results have important implications for public health experts to re-shape the emergency medical care system adapting it to the "new normal" while promoting better use of EDs and EMS.

\footnotetext{
Author contributions MP, AB, and AF contributed to the study conception and design. Material preparation, data collection, and analysis were performed by MP, CBA, MFV, AB, and MS. The first draft of the manuscript was written by MP, $\mathrm{AB}, \mathrm{MFV}$, and $\mathrm{CBA}$, and all authors commented on the following versions. All authors read and approved the final manuscript.
}

Funding The study did not receive any funding.

Data availability The datasets generated during and/or analyzed during the current study are available from the Azienda Zero-Veneto Region Health System's Clinical Governance Unit upon reasonable request.

Code availability Not applicable.

\section{Declarations}

Conflict of interest The authors declare that they have no conflict of interest.

Human and animal rights This retrospective study involving human participants was in accordance with the ethical standards of the institutional research committee and with the 1964 Helsinki Declaration and its later amendments or comparable ethical standards. The data used for this study was procured by the Veneto Regional Health System, which provided de-identified data. The ethical committee of the province of Vicenza, Veneto Region, approved this study (No. 96/20-October 13th, 2020).

Informed consent The informed consent was waived since the data collected were anonymous, as granted by the ethical committee authorization.

Consent to participate Since the data used for the study were deidentified and anonymous, the pertaining ethics committee ensured a waiver of consent as per the Italian and European laws on privacy and data protection.

Consent for publication Not applicable.

\section{References}

1. Keeley C, Jimenez J, Jackson H, Boudourakis L, Salway RJ, Cineas N, Villanueva Y, Bell D, Wallach AB, Boyle Schwartz D, Mendez-Justiniano I, Long TG (2020) Staffing up for the surge: expanding the New York city public hospital workforce during the COVID-19 pandemic. Health Aff (Millwood) 39(8):1426-1430. https://doi.org/10.1377/hlthaff.2020.00904

2. Emanuel EJ, Persad G, Upshur R, Thome B, Parker M, Glickman A, Zhang C, Boyle C, Smith M, Phillips JP (2020) Fair allocation of scarce medical resources in the time of COVID-19. N Engl J Med 382(21):2049-2055. https://doi.org/10.1056/NEJMsb2005 114

3. Paganini M, Conti A, Weinstein E, Della Corte F, Ragazzoni L (2020) Translating COVID-19 pandemic surge theory to practice in the emergency department: how to expand structure. Disaster Med Public Health Prep 14(4):541-550. https://doi.org/10.1017/ dmp. 2020.57

4. Man CY, Yeung RS, Chung JY, Cameron PA (2003) Impact of SARS on an emergency department in Hong Kong. Emerg Med (Fremantle) 15(5-6):418-422. https://doi.org/10.1046/j.14422026.2003.00495.x

5. Schanzer DL, Schwartz B (2013) Impact of seasonal and pandemic influenza on emergency department visits, 2003-2010, Ontario. Canada Acad Emerg Med 20(4):388-397. https://doi. org/10.1111/acem.12111

6. Santi L, Golinelli D, Tampieri A, Farina G, Greco M, Rosa S, Beleffi M, Biavati B, Campinoti F, Guerrini S, Ferrari R, Rucci P, Fantini MP, Giostra F (2021) Non-COVID-19 patients in times of pandemic: emergency department visits, hospitalizations and cause-specific mortality in Northern Italy. PLoS One 16(3):e0248995. https://doi.org/10.1371/journal.pone.0248995

7. Morello F, Bima P, Ferreri E, Chiarlo M, Balzaretti P, Tirabassi G, Petitti P, Aprà F, Vallino D, Carbone G, Pivetta EE, Lupia E (2021) After the first wave and beyond lockdown: long-lasting changes in emergency department visit number, characteristics, diagnoses, and hospital admissions. Intern Emerg Med 8:1-8. https://doi.org/10.1007/s11739-021-02667-2 
8. Raucci U, Musolino AM, Di Lallo D, Piga S, Barbieri MA, Pisani M, Rossi FP, Reale A, Ciofi Degli Atti ML, Villani A, Raponi M (2021) Impact of the COVID-19 pandemic on the emergency department of a tertiary children's hospital. Ital J Pediatr 47(1):21. https://doi.org/10.1186/s13052-021-00976-y

9. Desmond C, Brubaker KA, Ellner AL (2013) Decision-making strategies: ignored to the detriment of healthcare training and delivery? Health Psychol Behav Med 1(1):59-70. https://doi.org/ 10.1080/21642850.2013.854706

10. Wheaton MG, Prikhidko A, Messner GR (2021) Is fear of COVID19 contagious? The effects of emotion contagion and social media use on anxiety in response to the coronavirus pandemic. Front Psychol 5(11):567379. https://doi.org/10.3389/fpsyg.2020.567379

11. Ferry AV, Keanie C, Denvir MA, Mills NL, Strachan FE (2021) Chest pain presentations to hospital during the COVID-19 lockdown: lessons for public health media campaigns. PLoS One 16(4):e0249389. https://doi.org/10.1371/journal.pone.0249389

12. Buja A, Paganini M, Fusinato R, Cocchio S, Scioni M, Rebba V, Baldo V, Boccuzzo G (2021) Health and healthcare variables associated with Italy's excess mortality during the first wave of the COVID-19 pandemic: an ecological study. Medxriv. https:// doi.org/10.1101/2021.01.28.21250669

13. Stella F, Alexopoulos C, Scquizzato T, Zorzi A (2020) Impact of the COVID-19 outbreak on emergency medical system missions and emergency department visits in the Venice area. Eur J Emerg Med 27(4):298-300. https://doi.org/10.1097/MEJ.0000000000 000724

14. Paoli A, Brischigliaro L, Scquizzato T, Favaretto A, Spagna A (2020) Out-of-hospital cardiac arrest during the COVID-19 pandemic in the Province of Padua Northeast Italy. Resuscitation 154:47-49. https://doi.org/10.1016/j.resuscitation.2020.06.031

15. Moynihan R, Johansson M, Maybee A, Lang E, Légaré F (2020) Covid-19: an opportunity to reduce unnecessary healthcare. BMJ 370:m2752. https://doi.org/10.1136/bmj.m2752

Publisher's Note Springer Nature remains neutral with regard to jurisdictional claims in published maps and institutional affiliations. 